\title{
LA ESTRUCTURA MUSICAL EN LA NARRATIVA DE KATHERINE MANSFIELD Y JAMES JOYCE
}

\author{
KATHERINE MANSFIELD, JAMES JOYCE, MUSICAL STRUCTURE, \\ NARRATIVE, 2OTH CENTURY
}

María Jiménez Garcerán

Universidad de Murcia. Murcia, España maria.jimenez9@um.es

$\mathrm{D}$

ESDE FINALES del siglo XIX, y sobre todo a partir de la primera década del XX, es habitual que en la narrativa se desarrolle el tema del tiempo. En este trabajo, y según propone Baquero en Estructuras de la novela actual (1970), trato de hacer un análisis estructural comparativo del cuento "La lección de canto" de Katherine Mansfield y del capítulo once de Ulysses, de James Joyce. Ambas narraciones comparten una disposición musical de los diferentes textos que dialogan para formar el conjunto. Así, un análisis estructural ayudará a comprobar cómo la distribución de tipo musical está presente desde los formatos más breves hasta otros más extensos, hasta el punto en el que incluso se ha hablado de que toda Ulysses está ordenada según criterios musicales.

El libro Estructuras de la novela actual, con el que el profesor Mariano Baquero se incorpora a las teorías estructuralistas, es una historia de la novela moderna y contemporánea europea hasta 1970 a partir de las formas. A pesar de que, en ocasiones, la especificidad de ciertos moldes estructurales impone más límites que beneficios, considero que el estudio literario desde un punto de vista musical sí expande algunos horizontes de análisis: con suerte, horizontes interartísticos y, en muchas ocasiones, horizontes intertextuales como el que nos ocupa. En el capítulo VII, titulado "Estructura musical”, se estudian ciertas novelas modernas desde un enfoque de análisis textual proveniente de otro ámbito artístico. El lector quizá esté habituado a oír los términos sinfonía, contrapunto, leitmotiv, tema con variaciones, 
e incluso a usarlos metafóricamente para referirse a la literatura. Mariano Baquero, en este capítulo, sistematiza el uso de estos términos partiendo de su significado original y aplicándolo a determinadas obras literarias.

Según Baquero, el tema del tiempo, tan presente en el siglo XX, intenta convertir la novela en un arte del momento, como la música, y que el lector sienta el paso del tiempo, los cambios de tempo... Claudio Guillén llamó al tema del tiempo "una musicalización de la ficción". Además, el lector, en la novela moderna, se convierte en un ejecutante de la obra literaria, como los músicos de la partitura. De esta forma, parece lícito no sólo usar elementos y conceptos musicales para analizar el arte de novelar o introducir vocabulario musical en las narraciones, sino también la aplicación de estructuras musicales en la escritura de novelas o, desde el punto de vista del crítico, el estudio en términos musicales de la estructura de ciertas narraciones, como es el caso de este trabajo. En ese sentido, Baquero distingue entre estructuras musicales propiamente dichas (sinfonía, suite, tema con variaciones, fuga y contrapunto), el uso de leitmotiven y la asimilación de la estructura de la partitura a la de la página de la obra literaria. En este caso, para el análisis tanto de "La lección de canto" de Katherine Mansfield como del capítulo once de Ulysses de Joyce es muy útil la asimilación de la estructura contrapuntística, aunque en el estudio del capítulo de Joyce también introduciré el concepto de leitmotiv (Baquero, 1970).

¿Qué es el contrapunto? Para los no letrados en la materia, podría sonar más a matemáticas o física que a música. Incluso la antigua definición de "punto contra punto" o "nota contra nota" es exagerada: según Toch (2001 [1977]), es más adecuado definirlo sencillamente como punto de contraste. En una partitura, cuando dos entidades musicales se presentan al mismo tiempo, se tratan de forma contrapuntística con el objetivo de que suenen bien juntas. La naturaleza que relaciona esas dos entidades musicales es de oposición:

Las dos líneas musicales opuestas revelan las principales armas de la lucha, los dos medios principales de la expresión contrapuntística: el contraste en el ritmo y el contraste en la dirección del movimiento. Cuando una voz descansa, la otra avanza; cuando una asciende, la otra desciende.

Estas consideraciones dejan claro que, más que "nota contra nota", el contrapunto significa la presencia simultánea de dos (o más) voces, o 
líneas melódicas, como podríamos denominarlas, en movimiento contrastante (Toch, 2001 [1977]).

En el desarrollo de un pasaje contrapuntístico caben también momentos de descanso en esa lucha, en los que entre las dos voces "contradiciendo la contradicción", se disminuye la tensión. Ese juego de tensión y relajación es el que verdaderamente da forma al texto musical (Toch, 2001 [1977]). Para Mariano Baquero (1970), una novela puede tener estructura contrapuntística si cuenta "con la presencia de distintas fragmentadas historias que se van entrecruzando y sucediendo de acuerdo con tal disposición musical". Pone como ejemplo principal la novela Point counter Point, de Aldous Huxley, pero añade que "para Ralph Freedman el Ulysses de Joyce también 'is built on an obvious counterpoint"', aspecto que se puede comprobar, según Umberto Eco (apud Baquero, 1970) en el capítulo once de la novela: "el capítulo once, el de las Sirenas, con su estructura por analogías musicales, con el recurso de los temas narrativos y de los timbres sonoros, nos da una imagen contracta de la más amplia que reina en todo el volumen”. En este trabajo voy a aplicar el análisis contrapuntístico de la narrativa que propone Baquero a los dos textos citados.

Tanto en el cuento de Mansfield como en el capítulo del Ulysses encontramos una estructura contrapuntística compuesta por un elemento musical y otro narrativo, donde se da el juego de correspondencia-contraste entre la música y el pensamiento del protagonista. Lo característico de estos dos fragmentos es precisamente que una de las melodías del contrapunto sea musical, por la presencia de una fuente de sonido en la escena que presencia el personaje, y que sobre esa melodía se construyan el resto de líneas melódicas de los relatos. En lo que sigue, este trabajo está dividido en dos apartados principales, en los que, tras una contextualización se analizan, según las teorías expuestas, los dos textos citados. A continuación se establecerá la comparación entre ambos y la exposición de las conclusiones.

\section{La lección de canto}

Katherine Mansfield (1888-1923), nacida en Nueva Zelanda aunque vivió gran parte de su vida en Londres, concentró su labor literaria en la escritu- 
ra de cuentos: "sus cuentos, a fuerza de concisos y elementales, tomando un pequeño trozo de vida con apenas un poco de clímax argumental, vienen a ser a la vez el último ejemplo del sentido decimonónico de la narración y uno de los primeros ejemplos del sentido del nuevo siglo, en que una rebanada de tiempo vivido vale por sí solo sin necesidad de peripecia ni crisis de acción" (De Riquer y Valverde, 2010). Desde sus primeros cuentos se aprecian unas características recurrentes: abarcan poco tiempo en la vida de los protagonistas, se fijan en una situación mínima, la evolución de la acción es escasa, ejercen un profundo análisis de la psicología... En sus cuentos, como en sus diarios y cartas, se puede apreciar la relación que la autora tenía con la música, quien desde joven se integra en ambientes musicales e intenta desarrollar una carrera tocando el violonchelo (Katherine Mansfield Society, 2008). Esa afición se refleja en cuentos como "La lección de canto" (The singing lesson), que aparece integrado en la colección $L a$ fiesta en el jardín y otras historias (The garden party and other stories), de 1922.

\subsection{Análisis de la estructura}

"La lección de canto" es un cuento breve que relata apenas unos minutos de la clase de canto que imparte la señorita Meadows. Recientemente ha recibido una carta de su prometido en la que él rompe su compromiso. Esa carta atormenta a la señorita Meadows que, influida por sus sentimientos, pide a sus alumnas que canten los versos de la página catorce, sobre el paso del tiempo y el fin de la alegría. Mientras la señorita escucha la letra, no puede evitar acordarse de la carta de su prometido y reflexionar sobre ella. Cuando pide a las alumnas que traten de expresar mediante diferentes matices lo que dice la letra de la canción, ella lo hace con tanto sentimiento que prácticamente los hace llorar. En ese momento, una alumna le anuncia que la directora quiere verla: ha llegado un telegrama de su prometido, donde le pide que olvide la carta anterior. Cuando vuelve a clase, la señorita Meadows, rebosante de entusiasmo, pide a sus alumnas que canten la canción de la página treinta y dos, una celebración de la alegría.

Este cuento se presta a ser analizado desde un horizonte musical tanto por el uso de terminología propia de este ámbito como desde el punto de vista estructural. El primero de los puntos de vista, aunque no es el objetivo 
principal de este trabajo, incluye un manejo fluido y natural del repertorio léxico de los músicos. En ese sentido, destaca el párrafo en el que la señorita Meadows describe los giros que deben ejecutar sus alumnas para conseguir la expresión exigida por la letra de la canción:

Think of the words, girls. Use your imaginations. Fast! Ah, too Fast' cried Miss Meadows. 'That ought to break out -a loud, strong forte- a lament. And then in the second line, Winter Drear, make that Drear sound as if a cold wind were blowing through it. Dre-ear!' said she so awfully that Mary Beazley, on the music stool, wriggled her spine. 'The third line should be one crescendo. Fleetly! Ah, Fleetly Music's Gay Measure. Breaking on the first word of the last line, Passes. And then on the word, Away, you must begin to die ...to fade... until The Listening Ear is nothing more than a faint whisper ... You can slow down as much as you like almost on the last line (Mansfield, 1951 [1922]).

Por otro lado, se puede estudiar la estructura contrapuntística del cuento porque, en efecto, se combinan en él varios elementos que se desarrollan por contraste. La primera línea melódica que se introduce es la carta del prometido de la señorita Meadows, que es evocada por la maestra y aparece, asimismo, combinada con los pensamientos que provoca en ella. Frente a esta línea melódica se encuentran las canciones que la señorita Meadows hace cantar a sus alumnas. Si bien los sentimientos que provocan las cartas y los expresados por las canciones son similares, la tensión se establece por la alternancia entre dos sistemas diferentes: la letra de la canción, que el lector imagina como procedente de una fuente sonora externa -el piano y las niñas que cantan- y, por otro lado, los pensamientos de la profesora, que fluyen en su conciencia. Se puede decir que esos dos sistemas se retroalimentan: el estado de ánimo de la señorita Meadows la lleva a pedir la canción de la página catorce, al tiempo que es la canción la que provoca sus pensamientos negativos. Más adelante, cuando recibe el telegrama, cambia la canción como cambia el estado de ánimo de la protagonista.

Los dos textos que se combinan para formar este contrapunto aparecen primero presentados por completo, en la primera parte del cuento. Primero aparece el texto de la carta:

... I feel more and more strongly that our marriage would be a mistake. Not that I do not love you. I love you as much as it is possible for me to 
love any woman, but, truth to tell, I have come to the conclusion that I am not a marrying man, and the idea of settling down fills me with nothing but-' and the word 'disgust' was scratched out lightly and 'regret' written over the top (Mansfield, 1951 [1922]).

Y poco después aparece el texto de la canción:

Fast! Ah, too Fast Fade de Ro-o-ses of Pleasure;
Soon Autumn yields unto Wi-i-nter Drear,
Fleetly! Ah, Fleetly Mu-u-sic's Gay Measure
Passes away from the Listening Ear (Mansfield, 1951 [1922]).

Es a partir de entonces cuando empiezan a alternarse fragmentos de uno y otro texto:

Good Heavens, what could be more tragic than that lament” Every note was a sigh, a sob, a groan of awful mournfulness. Miss Meadows lifted het arms in the wide gown and began conducting with both hands. '... I feel more and more strongly that out marriage would be a mistake...' she beat. And the voices cried: Fleetly! Ah, Fleetly. What could have possessed him to write such a letter! What could have led up to it! (Mansfield, 1951 [1922]).

Como vemos, la técnica del fluir de la conciencia intenta reflejar la rapidez con que los pensamientos llegan a la mente de la protagonista, hasta tal punto que, en cierto momento, entre dos frases de la canción se inserta todo un párrafo del discurrir interior de la señorita Meadows:

Fast! Ah, too Fast. '... and the idea of settling was what he had written. That was a good as to say their engagement was definitely broken off. Broken off! Their engagement! People had been surprised enough that she had got engaged. The Science Mistress would not believe it at first. But nobody had been as surprised as she. She was thirty. Basis was twenty-five. It had been a miracle, simple a miracle, to hear him say, as the walked home from church that very dark night, 'You know, somehow or other, I've got fond of you.' And he had taken hold of the end of her ostrich feather boa. Passes away from the Listening Ear (Mansfield, 1951 [1922]). 
En la segunda parte del cuento, cuando la protagonista recibe el telegrama, la disposición de las líneas que conforman el contrapunto se repite: primero aparece el telegrama y, poco más adelante, la letra de la nueva canción. En los últimos párrafos son estas dos nuevas líneas melódicas las que se amalgaman.

\section{Capítulo once del Ulysses}

El Ulysses de Joyce se publicó en el mismo año que el cuento de Mansfield, en 1922. Además, el escritor dublinés y la autora de "La lección de canto" tienen en común una temprana afición por la música (Joyce estudió canto como tenor) y el reflejo de este interés en su literatura: Joyce tituló su primer libro de poemas Música de cámara, y "el gusto por el ritmo y por la música no haría desde entonces más que crecer en su obra” (De Riquer y Valverde, 2010). Su Ulysses narra unas veinte horas de la vida de Leopold Bloom, judío dublinés, y Stephen Dedalus, escritor, que ya aparecía en $R e$ trato de un artista adolescente. Martín de Riquer y José María Valverde definen el Ulysses como una combinación de dos alusiones simbólicas principales: a la Odisea y, como contrapunto -ellos mismos usan el término-, a la vida y la obra de Shakespeare. A estas se suman incontables referencias que han dado trabajo a numerosos hermeneutas. El estudio de la música en la obra de Joyce tampoco es algo nuevo: como curiosidad, se puede citar toda una página web dedicada a este tema (Music in the Works of James Joyce, 2003-2013) y el libro Musical Allusions in the Works of James Joyce: Early Poetry through Ulysses (1974), escrito por Zack Bowen.

\subsection{Análisis de la estructura}

Joyce emplea una técnica diferente en cada parte de la novela (De Riquer y Valverde, 2010). En el caso del capítulo 11, las sirenas, según las notas de la edición que del Ulysses hacen Don Gifford y Robert J. Seidman (2008), se trata de una Fuga per canone, es decir, una fuga de acuerdo con las normas habituales. Ellos analizan la estructura del capítulo como una fuga musical, con sus elementos habituales (sujeto, contrasujeto, respuesta...). Aunque se trata de un análisis correcto, no tiene tanto sentido en este trabajo como 
sí el estudio de secciones más reducidas, en las que se puede apreciar más claramente la técnica contrapuntística que he estado explicando. Además, de esta forma resulta más sencilla la comparación con el cuento breve de Mansfield.

Son las cuatro de la tarde y Leopold Bloom, en su recorrido por Dublín, llega al hotel Ormond, donde ha quedado para comer con Richie Goulding, tío de Stephen. Allí coincide con Blazes Boylan, el amante de Molly -esposa de Bloom-, que sale pronto del bar porque ha quedado con ella. Mientras escribe una carta a Martha, Leopold escucha las canciones que los otros clientes tocan al piano y contempla a las camareras, que cantan como las sirenas de la tradición épica. En toda esta escena priman los símbolos sonoros, los tecnicismos musicales y las referencias contextuales al arte del sonido. Se citan cantantes de la época (como Joseph Maas o Luigi Lablache; Gifford-Seidman, 2008), tecnicismos del ámbito musical (como Amoroso ma non troppo o da capo; Gifford-Seidman, 2008), fragmentos literarios que se refieren a la música (como "Music hath charms", de William Congreve, aunque atribuida a Shakespeare en este texto; Gifford-Seidman, 2008)... Junto a todas estas alusiones, cabe analizar un elemento que ya se acerca al análisis estructural: el leitmotiv. En música, consiste en el uso de temas o frases musicales determinadas asimiladas a personajes; se trata de un recurso habitual de la ópera. En el capítulo de Joyce, como en las grandes tragedias líricas, las primeras líneas están dedicadas a la presentación de los temas que después se desarrollarán: el capítulo comienza con una lista de motivos, ordenados según aparecen después en el texto. El uso del leitmotiv en música beneficia el modo como el espectador, a lo largo de la obra, admite los temas porque ya los conoce. En Joyce sucede algo similar con la relación de esas primeras líneas y el resto del capítulo.

No obstante, lo que más llama la atención es cómo en el capítulo coexisten, igual que en el cuento de Mansfield, diferentes líneas melódicas que se entrelazan mediante esa técnica que Baquero llamó contrapuntística. A lo largo del capítulo van apareciendo una serie de canciones, que son cantadas por los clientes y las camareras del bar, y que se integran después en el pensamiento de Bloom. Entre las recogidas por Gifford y Seidman se pueden contar hasta treinta y dos referencias musicales, entre las que destacan fragmentos de las óperas Floradora (1899; Gifford-Seidman, 2008) y $L a$ sonnambula (1831; Gifford-Seidman, 2008) y canciones como "Goodbye, 
Sweetheart, Goodbye" (Gifford-Seidman, 2008) o "The Last Rose of Summer" (Gifford-Seidman, 2008). Todas ellas aparecen tanto cantadas por los personajes como integradas en el discurrir mental del protagonista.

Por encima de todas estas referencias musicales destacan dos, que se imponen durante dos largas secuencias del capítulo. La primera es el aria que canta Lionel, personaje de la ópera Martha, de Flotow (GiffordSeidman, 2008). Se trata de una canción sobre el amor a primera vista y la exaltación del sentimiento amoroso del personaje hacia Martha. En el Ulysses de Joyce la canta Simon Dedalus a petición de Father Cowley. Los primeros versos del aria se van diseminando mientras Bloom, que va a escribir a su Martha, piensa en ella: en las primeras veces que se vieron, en sus encuentros, en su perfume. En el texto de Joyce la canción empieza a filtrarse progresivamente desde los guiones que introducen los versos que canta Dedalus hasta el pensamiento de Bloom. Por ejemplo:

-When first I saw that for endearing [...] Sorrow from me seemed to depart.

Through the hush of air a voice sang to them, low, not rain, not leaves in murmur, like no voice of strings of reeds or whatdoyoucallthem dulcimers, touching their still ears with words, still hearts of their each his remembered lives. Good, good to hear: sorrow from them each seemed to from both depart when first they heard. When first they saw (Joyce, 1969 [1922]).

Según la letra va ganando intensidad, las dos líneas melódicas -la canción y el pensamiento- se van integrando y, cuando Dedalus llega a la parte de la canción en que Lionel pronuncia el nombre de su amada, también Bloom está pensando en ella directamente: "Martha is it. Coincidence. Just going to write. Lionel's song. Lovely name you have". A partir de este momento, se va estrechando el espacio que separa la música de la mente de Bloom: los versos aparecen intercalados cada vez con más frecuencia en el fluir de su conciencia hasta que, al final de la canción, esta alternancia se combina en "Siopold": Simon que canta, el Lionel de la ópera y Leopold, al mismo tiempo.

La segunda referencia musical que cobra un protagonismo especial en el capítulo es la canción "The Croppy Boy" (Gifford-Seidman, 2008), que canta Ben Dollard más adelante. Su letra cuenta la historia de un mucha- 
cho que había perdido a su familia mientras buscaban la libertad. Antes de unirse a los rebeldes se confiesa, pero el sacerdote lo traiciona: es un militar disfrazado y lo hace ejecutar. En este caso el sincretismo entre la música y el discurrir mental de Bloom es tal que Joyce ni siquiera introduce los versos de la canción con guiones, sino que los integra directamente en el pensamiento del protagonista. Por ejemplo, si la canción dice "Is the priest at home? [...]/ The Priest's at home, boy, and may be seen:/ 'Tis easy speaking with Father Green;/ But you must wait, till I go and see/ If the holy father alone may be" (Gifford-Seidman, 2008: 293), Bloom piensa "The priest's at home. A false priest's servant bade him welcome. Step in. The holy father" (Joyce, 1969 [1922]). De esta forma se va integrando en la mente de Bloom toda la historia que cuenta la canción.

\section{Conclusiones y resultados}

Los dos fragmentos analizados son buenos ejemplos, a pequeña escala, de lo que Mariano Baquero había señalado como estructura contrapuntística en la novela. No obstante, hay que señalar que se trata de dos técnicas totalmente diferentes en cuanto a su complejidad. En el caso del cuento de Mansfield, la autora presenta primero cada uno de los temas para después combinarlos, aunque no tanto integrarlos, en el transcurso de los pensamientos de la protagonista. Sin embargo, Joyce, en línea con la dificultad de toda la novela, no expone previamente los temas, o al menos no de forma completa: las canciones aparecen dispersadas, fragmentadas, y después se integran en el pensamiento de Bloom de forma que el lector, en ocasiones, no nota que se trate de una referencia musical porque no todos los versos aparecen citados en cursiva. También es diferente la función que la música ostenta en cada obra. En "La lección de canto", la maestra elige la música según su estado de ánimo; es decir, el pensamiento es previo a la música, aunque resulta intensificado por causa de esta. Sin embargo, en el capítulo once del Ulysses de Joyce sucede al contrario: es la música la que marca el rumbo de la conciencia de Bloom en cada momento y, según él escucha las canciones, responde al estímulo con unas u otras reflexiones.

Creo que el análisis literario debería servirse más a menudo de este tipo de estructuras nacidas en los estudios de otras artes. Mediante el estudio de 
las dos narraciones desde la atalaya musical se ha comprobado que la literatura, a menudo considerada como un arte condicionado por la doble cara de su materia -significante y significado-, también puede usar recursos que la acerquen a otras artes. En este caso, la música aporta a la narración el factor temporal al que tanto valor le dan los narradores del siglo XX. El uso de la técnica contrapuntística, tanto en Mansfield como en Joyce, es un intento por imitar -yo diría que de forma hiperrealista- el acontecer natural de los hechos, que ocurren a la vez, y es precisamente el análisis en vertical, similar al estudio armónico de la partitura, el que demuestra cómo ambos autores consiguen que el lector así lo perciba.

\section{Referencias}

Baquero, M. (1970). Estructuras de la novela actual. Barcelona: Planeta.

De Riquer, M., Valverde, J. M. (2010). Historia de la literatura universal, II. Desde el Barroco hasta nuestros días. Tercera edición. Madrid: Gredos.

Gifford, D., Seidman, R. J. (eds.). (2008) Ulysses annotated. Segunda edición. Londres: University of California Press.

Joyce, J. (1969 [1922]). Ulysses. Middlesex: Penguin Books.

Katherine Mansfield Society (KMS). (2008). Katherine Mansfield Society. Disponible en http://www.katherinemansfieldsociety.org.

Mansfield, K. (1951 [1922]). The garden party. Middlesex: Penguin Books.

Toch, E. (2001 [1977]). Elementos constitutivos de la música. Armonía, melodía, contrapunto y forma. Barcelona: Idea Books. 